ARTICLE

Received 8 Sep 2016 | Accepted 13 Apr 2017 | Published 31 May $2017 \quad$ DOl: 10.1038/ncomms15654 OPEN

\title{
Oligolysine-based coating protects DNA nanostructures from low-salt denaturation and nuclease degradation
}

Nandhini Ponnuswamy 1,2,3, Maartje M.C. Bastings ${ }^{1,2,3}$, Bhavik Nathwani ${ }^{1,2,3}$, Ju Hee Ryu 1,2,3,4, Leo Y.T. Chou ${ }^{1,2,3}$, Mathias Vinther ${ }^{5}$, Weiwei Aileen Li ${ }^{3,6}$, Frances M. Anastassacos ${ }^{1,2,3}$, David J. Mooney ${ }^{3,6}$ \& William M. Shih ${ }^{1,2,3}$

DNA nanostructures have evoked great interest as potential therapeutics and diagnostics due to ease and robustness of programming their shapes, site-specific functionalizations and responsive behaviours. However, their utility in biological fluids can be compromised through denaturation induced by physiological salt concentrations and degradation mediated by nucleases. Here we demonstrate that DNA nanostructures coated by oligolysines to 0.5:1 $\mathrm{N}: \mathrm{P}$ (ratio of nitrogen in lysine to phosphorus in DNA), are stable in low salt and up to tenfold more resistant to DNase I digestion than when uncoated. Higher N:P ratios can lead to aggregation, but this can be circumvented by coating instead with an oligolysine-PEG copolymer, enabling up to a 1,000-fold protection against digestion by serum nucleases. Oligolysine-PEG-stabilized DNA nanostructures survive uptake into endosomal compartments and, in a mouse model, exhibit a modest increase in pharmacokinetic bioavailability. Thus, oligolysine-PEG is a one-step, structure-independent approach that provides low-cost and effective protection of DNA nanostructures for in vivo applications.

\footnotetext{
${ }^{1}$ Department of Cancer Biology, Dana-Farber Cancer Institute, 450 Brookline Avenue, Boston, Massachusetts 02215, USA. ${ }^{2}$ Department of Biological Chemistry and Molecular Pharmacology, Harvard Medical School, Boston, Massachusetts 02115, USA. ${ }^{3}$ Wyss Institute for Biologically Inspired Engineering at Harvard, Boston, Massachusetts 02115, USA. ${ }^{4}$ Center for Theragnosis, Biomedical Research Institute, Korea Institute of Science and Technology, Seoul 02792, Republic of Korea. ${ }^{5}$ Centre for DNA Nanotechnology, Interdisciplinary Nanoscience Center, iNANO, Aarhus University, Gustav Wieds Vej 14, 8000 Aarhus C, Denmark. ${ }^{6}$ School of Engineering and Applied Sciences, Harvard University, Cambridge Massachusetts 02138, USA. Correspondence and requests for materials should be addressed to W.M.S. (email: William_Shih@dfci.harvard.edu).
} 
$\mathrm{D}$ NA nanostructures (DNs) can be programmed to fold into prescribed spatial configurations ${ }^{1-6}$, functionalized site specifically with a wide variety of guests ${ }^{7}$ and engineered to undergo allosteric conformational changes ${ }^{8-11}$. For application to diagnostics and therapeutics, DNs can impart these useful properties, such as controlled shape, addressability and responsiveness, to other nanomaterials-for example, liposomes, polymeric or metallic particles, proteins-and thereby augment their functionality. Furthermore, DNs are biodegradable and biocompatible.

However, current biomedical applications of DNs are hindered by two main challenges. First, stabilization of most DNs requires $\sim 5-20 \mathrm{mM}$ divalent cations (for example, $\mathrm{Mg}^{2+}$ ) to overcome electrostatic repulsion between closely packed DNA phosphate anions. Therefore, most DNs exhibit poor structural integrity in physiological fluids, which typically contain low levels of $\mathrm{Mg}^{2+}$ and $\mathrm{Ca}^{2+}$ (for example, $\sim 0.4 \mathrm{mM}$ each in RPMI-1640) ${ }^{12}$. Second, nuclease activity results in rapid degradation during incubation of these structures in freshly prepared cell medium containing $10 \%$ fetal bovine serum (FBS) at $37^{\circ} \mathrm{C}^{13-16}$. Intravenous injection of a fluorescently labelled $\mathrm{DN}$ results in rapid clearance similar to that of a control oligonucleotide ${ }^{17}$. Thus, DNs must first be stabilized against $\mathrm{Mg}^{2}+$ depletion and nuclease degradation before they can be effective for most biomedical applications.

Several approaches have been investigated to address these challenges. Cassinelli et al. ${ }^{17}$ have demonstrated the use of click chemistry to catenate constituent tiles of six helix tubes, which renders these structures stable at low ionic strength; Benson et al. ${ }^{18}$ and Veneziano et al. ${ }^{19}$ have reported wireframe architectures for DNA origami that survive at physiological divalent cation concentrations. Coating of DNs with cowpea chlorotic mottle virus capsid proteins ${ }^{20}$ or with cationic poly(2-dimethylaminoethylmethacrylate) (PDMAEMA)-based polymers ${ }^{21}$ has been reported; however, this coating appeared to induce aggregation of DNs as monitored by negative-stain transmission electron microscopy (TEM). Our group has previously reported virus- inspired encapsulation of DN in liposomes, which protects the structure from nuclease degradation and enhances in vivo circulation time $\mathrm{e}^{22}$. However, this method requires attaching multiple lipid-DNA conjugates on DNs at defined positions and extensive protocol optimization to template bilayer self-assembly without aggregation or multimerizaton, which makes the process time consuming. Although successful in addressing individual challenges, each of these methods hence remain labour/cost intensive or are structure dependent. A need therefore remains for an easy, scalable and structure-independent method to stabilize DNs against commonly encountered in vivo threats.

We report here that coating DNs with oligolysine provides stability against denaturation at physiological $\mathrm{Mg}^{2}+$ concentrations without noticeable distortion or aggregation of the structure (as monitored by negative-stain TEM). Coating is driven by electrostatic interactions and is achieved simply by mixing stock solutions of DNs and oligolysine at appropriate stoichiometric ratios followed by incubation at room temperature. However, coating with oligolysine only modestly protects DNs against nucleases. Refining our approach, we show that coating with oligolysine conjugated to polyethylene glycol (PEG) significantly slows down nuclease degradation. Furthermore, we use a fluorescence resonance energy transfer (FRET)-based assay to demonstrate structural integrity of the coated nanostructures within cellular compartments.

\section{Results}

Stabilization of DNs against low-salt denaturation. We hypothesized that electrostatic repulsions in DNs due to $\mathrm{Mg}^{2+}$ depletion could be stabilized by using polyamines as a substitute for divalent cations (schematic with oligolysines as stabilizing agent shown in Fig. 1), as they would remain bound after dilution into physiological buffers (for example, see Supplementary Table 1 for RPMI-1640 composition), due to their much stronger binding. However, polyamines are known to condense DNA into compact nanoparticles; therefore, one concern is that

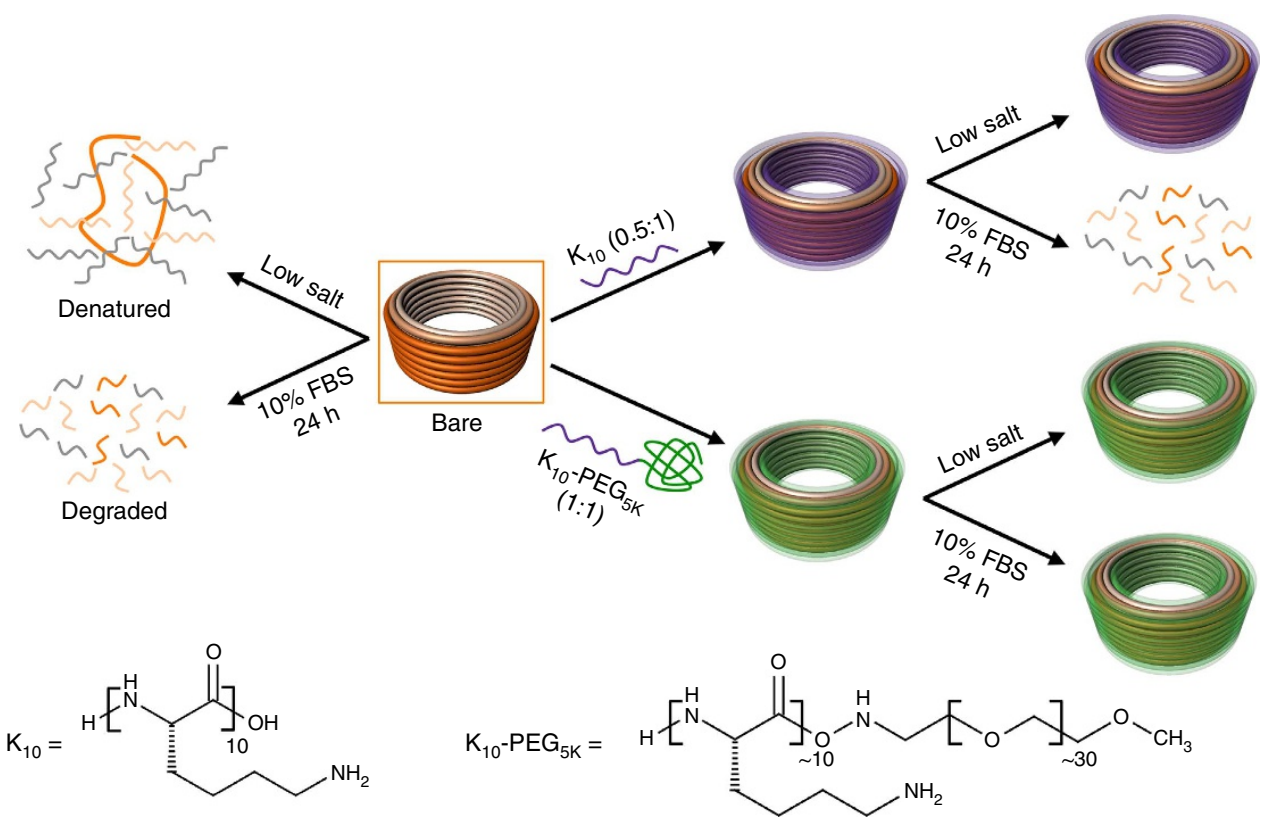

Figure 1 | Protection of DNs from low salt and nucleases. The schematic represents the fate of bare and coated DNs in physiological buffers at $37^{\circ} \mathrm{C}$ containing low salt and/or 10\% FBS. Bare DN rapidly denatures at low salt and degrades in freshly prepared cell medium containing $10 \%$ FBS. Low-salt-induced denaturation and nuclease degradation can be overcome by coating the DNs with positively charged peptides such as $\mathrm{K}_{10}$ or $\mathrm{K}_{10}-\mathrm{PEG}_{5 \mathrm{~K}}$ at an $\mathrm{N}: \mathrm{P}$ of $0.5: 1$ and 1:1, respectively. The oligolysine peptide has $\alpha$-lysine side chains. 


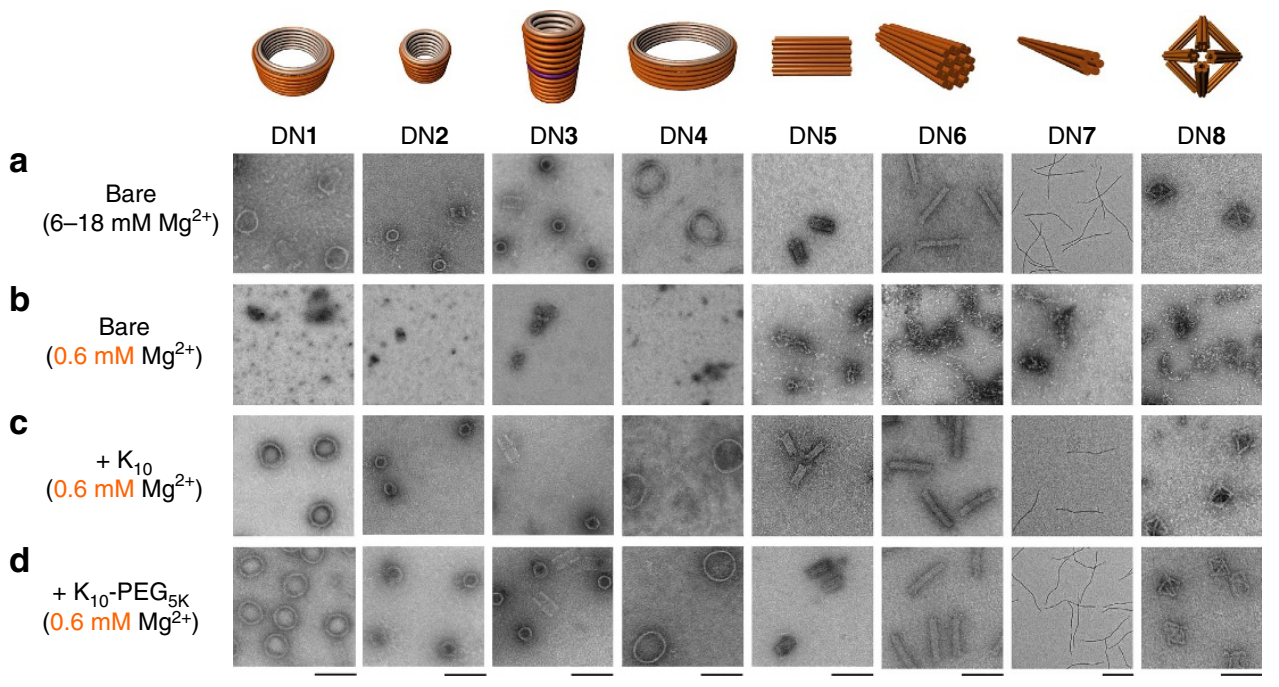

Figure 2 | Low-magnesium stability of bare and coated DNs. (a) Negative-stain TEM images of bare DNs DN1-DN8 in folding buffer (5 mM Tris, $1 \mathrm{mM}$ EDTA, 6-18 $\mathrm{mM} \mathrm{Mg}^{2+}$ ). Negative-stain TEM images of (b) bare DN1-DN8, (c) K10-coated DN1-DN8, (d) K 10 -PEG5 $\mathrm{K}_{\mathrm{K}}$-Coated DN1-DN8 diluted in physiological buffer (RPMI-1640) such that the final $\mathrm{Mg}^{2+}$ concentration is $\sim 0.6 \mathrm{mM}$. All scale bars are $100 \mathrm{~nm}$, except for DN7 (200 nm).

polyamine binding could crush or deform DNs and thereby compromise their intricate three-dimensional shapes important for their functionality. A second concern is that polyamine binding could lead to aggregation of DNs.

We first evaluated the structural integrity at low $\mathrm{Mg}^{2}+$ of a model DN that has the shape of a barrel (DN1 in Fig. 2) using a set of various polyamines: spermidine, spermine, oligoarginines of length 6, oligolysines of length 6 and $10 \mathrm{kDa}$ branched polyethyleneimine (PEI). All the polyamines are positively charged at physiological $\mathrm{pH}$ and have the ability to interact with DNA electrostatically (Supplementary Fig. 1). Coating of DN1 was achieved by mixing DN1 with a solution of the polyamine at the desired N:P ratio (nitrogen in amines to phosphorus in DNA) and incubating at room temperature for $30 \mathrm{~min}$. To assess the structural integrity of DN1 in physiological buffers, bare and polyamine-coated DN1 were diluted in standard RPMI medium $\left(133 \mathrm{mM} \mathrm{Na}^{+}, 5.3 \mathrm{mM} \mathrm{K}^{+}, 0.4 \mathrm{mM} \mathrm{Mg}^{2+}\right.$ and $\left.0.4 \mathrm{mM} \mathrm{Ca}^{2+}\right)$ such that the final $\mathrm{Mg}^{2+}$ concentration was $\sim 0.6 \mathrm{mM}$. The solution was then heated at $37^{\circ} \mathrm{C}$ for $1 \mathrm{~h}$ in a thermal cycler and then analysed using agarose gel electrophoresis (AGE) and TEM.

Analysis of the bare DN1 at physiological levels of $\mathrm{Mg}^{2+}$ ions revealed that its structure was destroyed beyond recognition (assessed via TEM, Fig. 2) and with dissociation of staple strands from the scaffold strand (assessed via AGE, Supplementary Fig. 2). Structural integrity at low salt could be preserved by prior incubation with spermine and spermidine, but only at $\mathrm{N}: \mathrm{P}$ ratios of 50:1 and 100:1, respectively. However, these samples denatured after overnight dialysis at room temperature into zero- $\mathrm{Mg}^{2+}$ buffer ( $5 \mathrm{mM}$ Tris, $1 \mathrm{mM}$ EDTA $\mathrm{pH} 8.0$ ), presumably due to dissociation of the polyamines into the free solution. This result encouraged us to try longer polyamines to obtain stronger binding and therefore longer residence times, as well as better control over bound N:P ratios. Pre-incubation of DN1 with PEI led to destruction of structural integrity due to compaction as observed by TEM, likely to be caused by strong multivalent binding (Supplementary Figs 2 and 3). In contrast, pre-incubation with oligoarginines protected against low-salt denaturation, however, at the cost of significant structural deformation (that is, barrel projection takes the appearance of a polygon instead of a circle (Supplementary Fig. 3).

We obtained the best results by far with oligolysine-coated DN1, which withstood divalent cation depletion at $37^{\circ} \mathrm{C}$ and maintained structural integrity as observed via TEM. Different lengths of oligolysines $\mathrm{K}_{n}$ (where $n$ is the number of L-lysine monomers; $n=4,6,8,10,12,16$ and 20) were investigated (Supplementary Figs 4-6). We observed that shorter oligolysines $(n<10)$ required high N:P ratio for stabilization of DNs, owing to their weaker binding. Longer oligolysines $(n>10)$, on the other hand, were required in only sub-stoichiometric amounts for stabilization due to enhanced binding, however, at the cost of increased aggregation of DNs. The best results were observed for $\mathrm{K}_{10}$, where the preservation of structure could be achieved at an $\mathrm{N}: \mathrm{P}$ ratio of 0.5:1. Some aggregates were observed by TEM and AGE, but most of these separated after the sample was diluted to $1 \mathrm{nM}$ in RPMI- 1640 and incubated at $37^{\circ} \mathrm{C}$ for $1 \mathrm{~h}$.

The stability induced by $\mathrm{K}_{10}$ at low divalent-cation concentration was further tested with other DN designs (labelled DN2-DN8; Fig. 2). These nanostructures were chosen as a representative set to highlight the generality of the approach against variations in structural features such as curvature, the presence of a cavity, charge density, crossover density and size (AGE and TEM analysis in Supplementary Figs 7 and 8). The unstabilized DNs required 6-18 $\mathrm{mM} \mathrm{Mg}^{2+}$ to maintain their intact structures. In all the cases, upon dilution in standard RPMI medium, the bare structure denatured to scaffold and staple strands, as observed via AGE and TEM. In contrast, DNs coated with $\mathrm{K}_{10}$ were able to maintain complete structural integrity even after overnight dialysis into zero$\mathrm{Mg}^{2+}$ buffer ( $5 \mathrm{mM}$ Tris, $1 \mathrm{mM}$ EDTA pH 8.0; Supplementary Figs 9-13).

Stability of DNs against nuclease degradation. Encouraged by our results towards $\mathrm{Mg}^{2+}$ depletion, we moved on to tackle the second main challenge, which was to prevent rapid nuclease degradation of DNs in serum. Previous studies have reported that positively charged polyamines can condense DNA into nanoparticles that are protected from digestion by nucleases, a behaviour that has been exploited extensively in gene-delivery applications ${ }^{23}$. Therefore, we next investigated whether $\mathrm{K}_{10}$ coating protects DNs from nuclease digestion, despite not crushing them into condensed particles. For this and subsequent experiments, we focused on DN1 as a model system; we selected DN1 as a challenging test case for nuclease resistance, as it has very high surface area to mass ratio. 

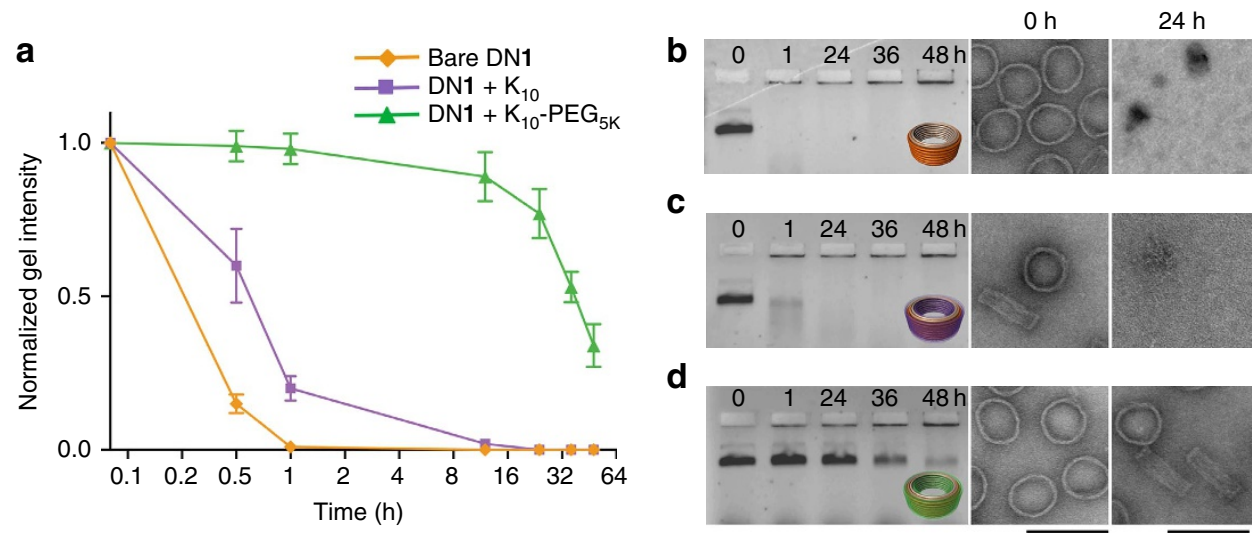

Figure 3 | Time course of nuclease degradation of bare and coated DN1. Digestion was assayed in freshly prepared cell medium (RPMI-1640 containing $10 \% \mathrm{FBS}$ ) at $37^{\circ} \mathrm{C}$. (a) Normalized agarose gel intensity vs. time for bare, $\mathrm{K}_{10}$ coated and $\mathrm{K}_{10}-\mathrm{PEG}_{5 \mathrm{~K}}$-coated DN1. Time (hours) is plotted with $\log _{2}$ scaling and error bars represent s.d. $(n=3 \mathrm{~s})$. Agarose gel and negative-stain TEM images at $\mathrm{O}$ h and $24 \mathrm{~h}$ for $(\mathbf{b})$ bare DN1, (c) $\mathrm{K}_{10}-\mathrm{coated} \mathrm{DN} \mathbf{1}$ and (d) $\mathrm{K}_{10}-\mathrm{PEG}_{5 \mathrm{~K}}$-coated DN1 incubated in freshly prepared primary BMDC medium at $37^{\circ} \mathrm{C}$. Scale bars, $100 \mathrm{~nm}$.

To assess the kinetics of nuclease digestion, the bare and $\mathrm{K}_{10}$ $(\mathrm{N}: \mathrm{P}=0.5: 1)$-coated $\mathrm{DN} 1$ were incubated in RPMI supplemented with $10 \% \mathrm{FBS}$ at $37^{\circ} \mathrm{C}$ for different time points. After incubation, the samples were analysed by AGE and TEM, and compared with a control incubated in RPMI $+10 \mathrm{mM} \mathrm{Mg}^{2+}$, but lacking FBS. Bare DN1 reached $50 \%$ degradation by $\sim 5 \mathrm{~min}$ in standard RPMI buffer, whereas it did not reach $50 \%$ degradation until $\sim 55 \mathrm{~min}$ in RPMI supplemented with $10 \mathrm{mM} \mathrm{Mg}^{2+}$. Likewise, $\mathrm{K}_{10}$-coated DN1 (N:P=0.5:1) did not reach $50 \%$ degradation until $\sim 50 \mathrm{~min}$. Therefore, the $\mathrm{K}_{10}$ treatment protects DN1 to the same degree $(\sim 5-10$-fold enhanced resistance to nuclease degradation) as $\mathrm{Mg}^{2+}$ (Fig. 3). We hypothesize this is primarily due to the preservation of overall structure, and that low-salt denatured structures are better substrates for serum nucleases, perhaps due to their more open and accessible configurations (Supplementary Figs 14-17).

Seeking further improved resistance against nuclease digestion, we explored the use of a $\mathrm{K}_{10}-\mathrm{PEG}_{n \mathrm{~K}}$ co-polymer where the amino terminus of $\mathrm{K}_{10}$ was covalently functionalized with either a 1,5 or $20 \mathrm{~K} \mathrm{PEG}_{n}(n=1,5$ and $20 \mathrm{~K})$. PEGylation of $\mathrm{K}_{10}$ was chosen because previous studies have reported an improvement in stability and increase in vivo circulation times post PEGylation of nanoparticles ${ }^{24}$. Furthermore, PEGylation might discourage aggregation of particles and therefore enable the use of higher $\mathrm{N}: \mathrm{P}$ ratios that otherwise would lead to such aggregation yet might provide greater nuclease resistance. Coating of DN1 with $\mathrm{K}_{10}-\mathrm{PEG}_{n \mathrm{~K}}$ was performed using the same protocol as described above for $K_{10}$, but with a N:P ratio of $1: 1$.

$\mathrm{K}_{10}-\mathrm{PEG}_{n \mathrm{~K}}$-coated DN1 was imaged via TEM, to ensure that the dense coating of $\mathrm{K}_{10}-\mathrm{PEG}_{n \mathrm{~K}}$ did not compromise structural integrity in low-salt physiological buffers. TEM images showed that DN1 coated with $\mathrm{K}_{10}-\mathrm{PEG}_{5 \mathrm{~K}}$ or $20 \mathrm{~K}$ at a $\mathrm{N}: \mathrm{P}$ ratio of $1: 1$ were intact and displayed no visible difference when compared with the bare nanostructures (Supplementary Fig. 18). In contrast, DN1 coated with $\mathrm{K}_{10}-\mathrm{PEG}_{1 \mathrm{~K}}$ at a $\mathrm{N}: \mathrm{P}$ ratio of $1: 1$ were extensively aggregated (Supplementary Fig. 18). The $\mathrm{K}_{10}-\mathrm{PEG}_{n \mathrm{~K}}$-coated DNs are neutral at a N:P ratio of $1: 1$ and hence are retained in the well during AGE. Before AGE, any $\mathrm{K}_{10}-\mathrm{PEG}_{n \mathrm{~K}}$ coating can be removed if desired by incubating the sample with chondroitin sulfate, a negatively charged sulfated glycosaminoglycan, which electrostatically sequesters the positively charged $\mathrm{K}_{10}-\mathrm{PEG}_{n \mathrm{~K}}$ and allows the DNs to penetrate the gel matrix (Supplementary Fig. 9).

To assess whether these PEGylated oligolysine coatings would provide greater resistance against nuclease degradation, we incubated the $\mathrm{K}_{10}-\mathrm{PEG}_{5 \mathrm{~K}}$ or $20 \mathrm{~K}^{-}$coated DN1 (1 nM) in RPMI supplemented with $10 \% \mathrm{FBS}$ at $37^{\circ} \mathrm{C}$ for different time points. Before AGE, nucleases can be deactivated using $5 \mathrm{mM}$ EGTA and $10 \% \quad \beta$-mercaptoethanol ${ }^{25}$ (Supplementary Fig. 19), and subsequently oligolysine shell can be removed by incubation with chondroitin sulfate to enable AGE analysis. Strikingly, AGE and TEM analysis showed that $\mathrm{K}_{10}-\mathrm{PEG}_{5 \mathrm{~K}}$-coated $\mathrm{DN1}$ reached $50 \%$ degradation in $\sim 36 \mathrm{~h}$ compared with only $5 \mathrm{~min}$ for the bare DN1, corresponding to a $\sim 800 \times$ enhancement in resistance to nuclease degradation (Fig. 3). $\mathrm{K}_{10}-\mathrm{PEG}_{20 \mathrm{~K}}$-coated DN1, on the other hand, showed almost comparable nuclease protection (50\% degradation at $\sim 24 \mathrm{~h}$ ) compared with $\mathrm{K}_{10}-\mathrm{PEG}_{5 \mathrm{~K}}$-coated DN1 (Supplementary Fig. 20).

We also performed a DNase I titration assay, where bare and $\mathrm{K}_{10}-\mathrm{PEG}_{5 \mathrm{~K}}$-coated DN1 $(1 \mathrm{nM})$ were incubated with increasing amounts of DNase I in standard RPMI medium for $1 \mathrm{~h}$ at $37^{\circ} \mathrm{C}$. Bare DN1 was $\sim 50 \%$ degraded in $\sim 0.5 \mathrm{U} \mathrm{ml}^{-1}$ of DNase I, as observed by AGE. In contrast, $\mathrm{K}_{10}-\mathrm{PEG}_{5 \mathrm{~K}-}$ coated DN1 did not show significant degradation even in $\sim 500 \mathrm{U} \mathrm{ml}^{-1}$ of DNase I as observed by AGE and TEM, corresponding to a $\sim 1,000$-fold increase in nuclease resistance (Supplementary Figs 21 and 22).

DNs such as DN9 (mesh helix ${ }^{19}$; Supplementary Fig. 23), constructed using a polyhedral mesh architecture, are intact in low-salt buffers due to the low incidence of adjacent double helices and therefore exhibit greater nuclease resistance at low salt compared with multilayer DNs such as DN1 (ref. 19). We also found that even DN9 exhibit $\sim$ fivefold greater nuclease resistance when coated with $\mathrm{K}_{10}-\mathrm{PEG}_{5 \mathrm{~K}}$ (Supplementary Fig. 24). This result suggests that the nuclease resistance offered by $\mathrm{K}_{10}-\mathrm{PEG}_{5 \mathrm{~K}}$ is not only due to the preservation of structural integrity of DNs in low-salt buffers, but also due in part to other factors such as steric shielding provided by the PEG layer that may reduce binding of nucleases to the DNA.

Accessibility of surface receptors in $\mathrm{K}_{10}-\mathrm{PEG}_{5 \mathrm{~K}}$-coated DNs. Next we addressed whether $\mathrm{K}_{10}-\mathrm{PEG}_{5 \mathrm{~K}}$ coating of DNs blocks addressable surface features from functionalization (the attachment of $5 \mathrm{~K}$ PEG at a N:P of 1:1 increases the thickness of the nanostructure by $2.4 \pm 1.3 \mathrm{~nm}$ as measured by dynamic light scattering); as a proxy, we assayed the ability of single-stranded DNA (ssDNA) handles protruding of the surface of DNs to interact with ssDNA anti-handles in solution. For this purpose, we incorporated 12 inward-facing 21mer ssDNA handles on the inside of DN1, to be bound by Alexa-750-labelled DNA anti-handles recruited from bulk solution (Supplementary Figs 25 and 26). We incubated 


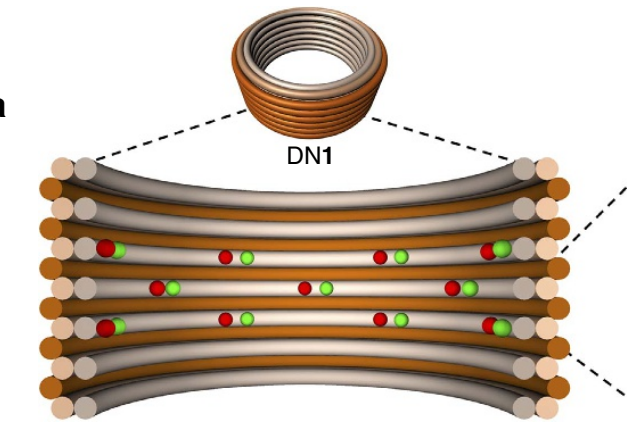

b
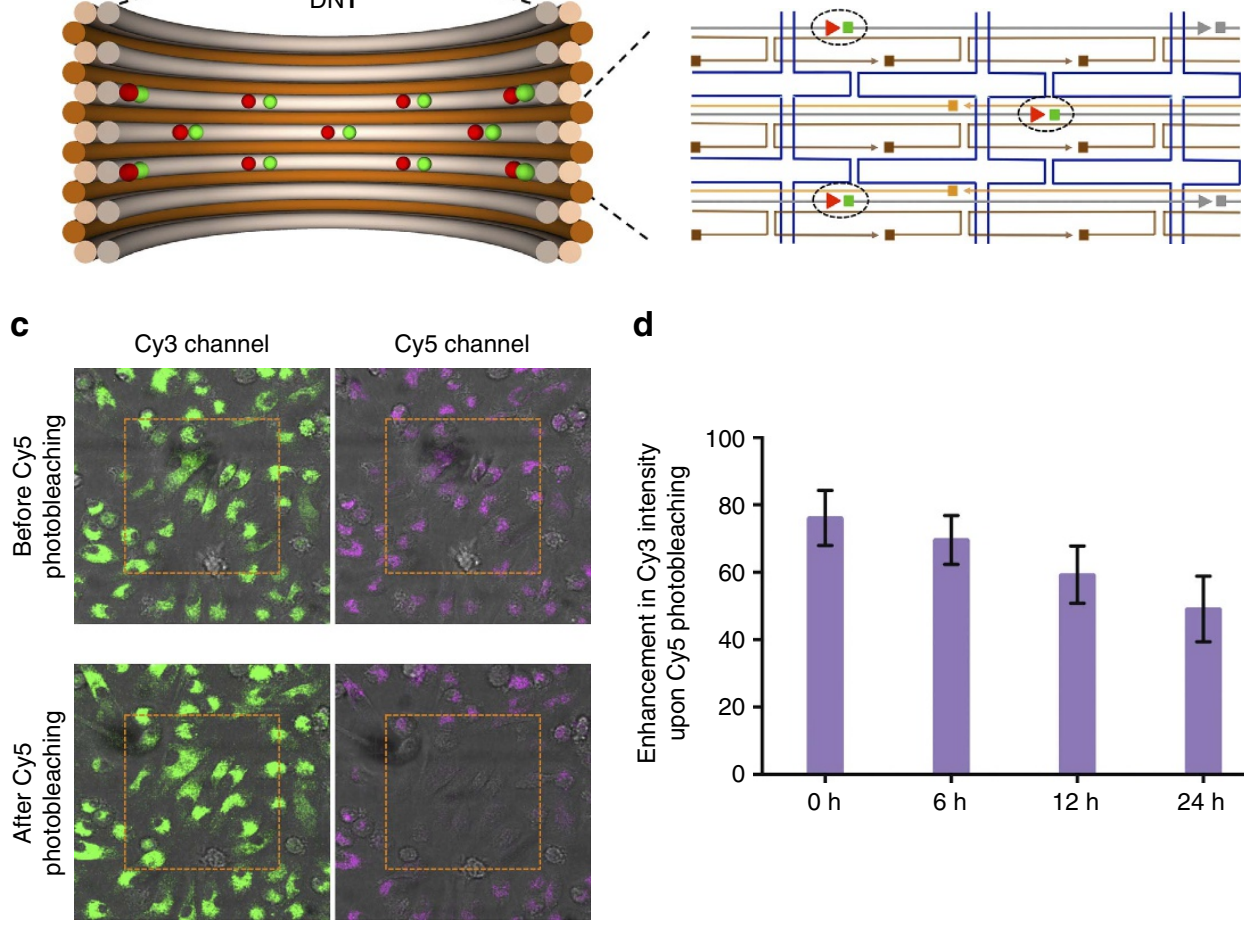

d

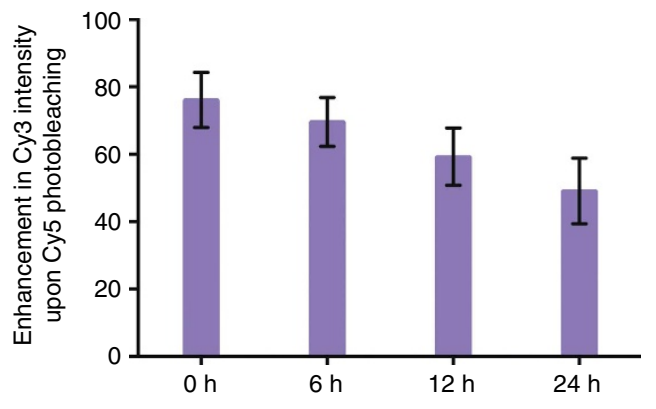

Figure 4 | FRET assay of intracellular integrity of $\mathbf{K}_{\mathbf{1 0}}-\mathbf{P E G}_{\mathbf{5 K}}$ coated DN1. (a) Schematic representation of the cross section of DN1 functionalized with Cy3 (green) and Cy5 (red) fluorophores. (b) caDNAno ${ }^{32}$ diagram highlighting the position of fluorophores relative to the other staples (blue: p7308 scaffold; brown: outer staples; orange: inner-middle staples; grey: inner staples) (c) Confocal images of primary BMDCs from mice incubated with Cy3 and Cy5 fluorophore labelled DN1. (d) Enhancement in Cy3 intensity upon Cy5 photobleaching of BMDCs measured using confocal microscopy at 0, 6, 12 and 24 normalized to in vitro measurements. Error bars represent s.d. $(n=2)$.

DN1 containing the ssDNA handles, bare versus $\mathrm{K}_{10}$-coated versus $\mathrm{K}_{10}-\mathrm{PEG}_{5 \mathrm{~K}}$-coated, with Alexa-750-labelled DNA anti-handle for $2 \mathrm{~h}$ at $37^{\circ} \mathrm{C}$ and monitored the incorporation of the Alexa 750 fluorophore into DN1 using AGE. We observed that the incorporation efficiency of Alexa 750 was almost identical in all the three cases, implying that $\mathrm{K}_{10}-\mathrm{PEG}_{5 \mathrm{~K}}$ coating of $\mathrm{DNs}$ allows accessibility to surface decorations.

To further demonstrate that targeting by receptors positioned on DNs is not inhibited by the $\mathrm{K}_{10}-\mathrm{PEG}_{5 \mathrm{~K}}$ coating, we positioned 72 $\alpha v \beta 3$-integrin-specific cyclic arginine-glycine-aspartate ${ }^{26}$ ligands strategically on the surface of DN6. We coated DN6 with $\mathrm{K}_{10^{-}}$ $\mathrm{PEG}_{5 \mathrm{~K}}$ and monitored cellular uptake into human umbilical vein endothelial cells by flow cytometry analysis. Our data shows that we are able to target surface integrin receptors on the cells and modestly enhance cellular uptake (Supplementary Fig. 27).

Cellular uptake of $\mathrm{K}_{10}-\mathrm{PEG}_{\mathbf{5 K}}$-coated DN1 into BMDCs. Thus far, we had demonstrated that coating of DNs with $\mathrm{K}_{10}-\mathrm{PEG}_{5 \mathrm{~K}}$ at an $\mathrm{N}: \mathrm{P}$ ratio of $1: 1$ stabilizes them against $\mathrm{Mg}^{2+}$ depletion and nuclease degradation. Towards cellular and downstream in vivo applications, we next investigated whether $\mathrm{K}_{10}-\mathrm{PEG}_{5 \mathrm{~K}}$ coating is non-toxic and allows cellular uptake of DNs. For the subsequent experiments, we designated uncoated $\mathrm{DN1}$ as $\mathrm{DN1} \mathbf{1}_{\text {bare }}$ and $\mathrm{K}_{10}-\mathrm{PEG}_{5 \mathrm{~K}}$-coated DN1 as DN1 $1_{\text {coated }}$.

We chose to observe the cellular uptake of $\mathrm{DN1} \mathbf{1}_{\text {bare }}$ and $\mathrm{DN1} 1_{\text {coated }}$ into mouse primary bone marrow-derived dendritic cells (BMDCs). BMDCs provide a challenging test case, as they have been reported to degrade endocytosed DNA with great efficiency ${ }^{27}$. We incorporated either $18 \mathrm{Cy} 5$ or $18 \mathrm{Cy} 3$ fluorophores into the inner DNA helices of DN1. The fluorophorelabelled DN1 was incubated with BMDCs for $12 \mathrm{~h}$ and visualized using confocal fluorescence microscopy.

As expected, $\mathrm{DN1}_{\text {bare }}$ was not taken into the cells to a measurable extent; we infer that this is partly due to its rapid denaturation and degradation in the culture medium (Supplementary Fig. 28). In contrast, DN1 $1_{\text {coated }}$ was internalized into dendritic cells (Supplementary Fig. 29) and, most importantly, we did not observe any measurable cytotoxicity (that is, signs of apoptosis) at

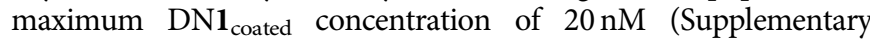
Note 1 and Supplementary Figs 49 and 50). BMDCs incubated with $\mathrm{DN1} \mathbf{1}_{\text {coated }}$ exhibited punctate fluorescence indicative of DNs trapped in vesicles. Subsequently, we co-incubated BMDCs with Cy5-labelled DN1 $1_{\text {coated }}$ and fluorescein-labelled transferrin, which is a marker for endosomal compartments. Similar punctate fluorescence was observed in both colour channels, suggesting that DNs were mostly resident within endosomal and endolysosomal compartments of cells (Supplementary Fig. 30).

Structural integrity of DN inside cell compartments. The environment of cellular compartments can be drastically different from the outside medium and, therefore, it is of great interest to investigate the persistence and structural integrity of DNs within those compartments. To monitor the persistence of DNs within endosomal compartments of cells, we incubated Cy5-labelled DN1 $1_{\text {coated }}(5 \mathrm{nM}$ final DN1 concentration, $\sim 90 \mathrm{nM}$ Cy5 fluorophore) with BMDCs for $12 \mathrm{~h}$ and then washed the cells with 
a

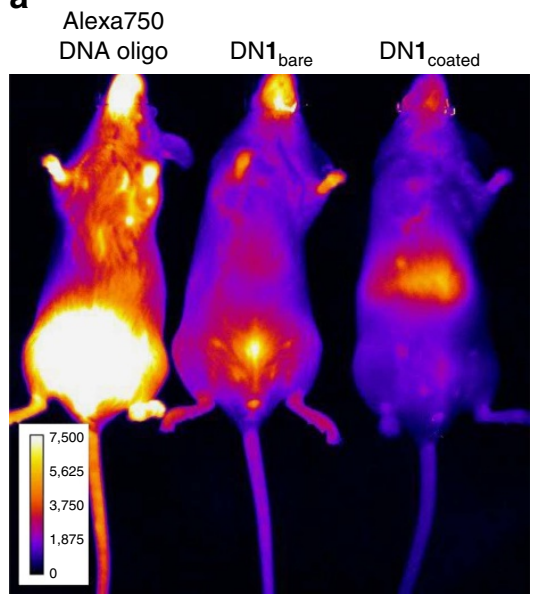

b

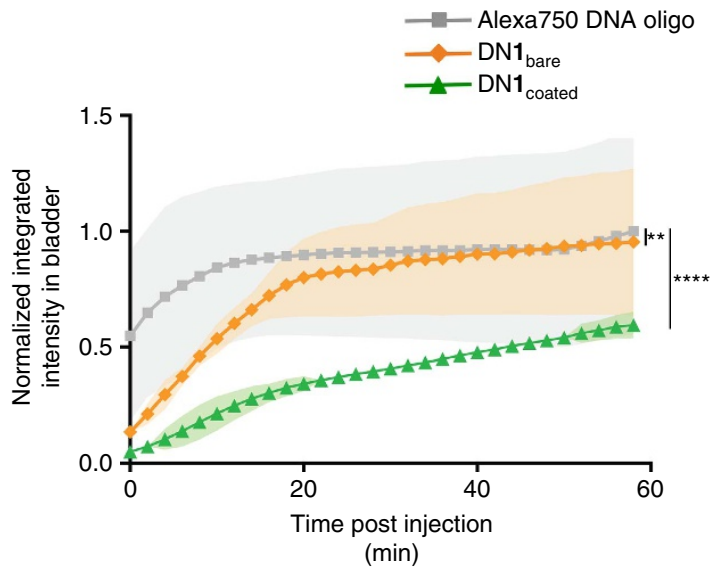

Figure 5 | In vivo optical imaging for biodistribution and pharmacokinetics of DN1 $\mathbf{1}_{\text {coated }}$ (a) Fluorescence images of mice injected with either Alexa750 DNA oligo or DN1 $\mathbf{1}_{\text {bare }}$ and $\mathrm{DN} \mathbf{1}_{\text {coated }}$ functionalized with 36 Alexa750 fluorophores at 2 min post injection. (b) Mean fluorescence intensity in bladder versus time. (Shaded regions represent s.d., $n=3$; two-way analysis of variance test; ${ }^{\star \star} P<=0.01$ and ${ }^{\star \star \star \star} P<0.0001$ ).

culture medium to remove any excess DNs present in the medium. Subsequently, we monitored the decay in fluorescence intensity within cells for $24 \mathrm{~h}$. We observed that fluorescence from DN1 $\mathbf{1}_{\text {coated }}$ displayed a half-life of $\sim 8 \mathrm{~h}$ post washing within cells. DNs either can be exported as intact structures or else first degraded and then exported; our experiments could not differentiate between these two export trajectories (Supplementary Fig. 31).

To further assess the structural integrity of DNs within cells, we designed a FRET assay based on donor-fluorescence enhancement induced by acceptor photobleaching ${ }^{28}$; this is a commonly used technique to enhance the sensitivity of FRET detection. For this purpose, every alternate DNA oligonucleotide binding to the inner DNA helices (Fig. 4) of DN1 $\mathbf{1}_{\text {coated }}$ was either functionalized at its $3^{\prime}$-end with an acceptor Cy5 fluorophore or at its $5^{\prime}$-end with a donor Cy3 fluorophore. The design is such that in an intact structure, the distance between adjacent Cy3 and Cy5 fluorophores is well under the estimated Förster distance of $\sim 5.6 \mathrm{~nm}$; the distance between non-adjacent fluorophores is $\sim 8 \mathrm{~nm}$. Energy transfer from a $\mathrm{Cy} 3$ donor to its adjacent Cy5 acceptor will result in the quenching of Cy3 fluorescence. After all Cy5 acceptors are photobleached using strong laser intensity, energy transfer from Cy 3 to $\mathrm{Cy} 5$ is destroyed, resulting in the effective enhancement of the $\mathrm{Cy} 3$ donor signal. Alternatively, for structures where the close arrangement between $\mathrm{Cy} 3$ to $\mathrm{Cy} 5$ had previously been disrupted due to prior denaturation and/or degradation, then photobleaching of Cy5 will not result in any further $\mathrm{Cy} 3$ fluorescence enhancement. In cells, the amount of $\mathrm{Cy} 3$ fluorescence enhancement due to photobleaching therefore can be used to infer the amount of intact structures within the cells.

To validate that our assay could be used to monitor structural integrity, first we verified the experiment on a single-molecule level in a cell-free environment by total internal reflectance fluorescence (TIRF) microscopy. For these measurements, Cy3/ Cy5-labelled DN1 $1_{\text {coated }}$ was functionalized with 12 biotins and deposited on a streptavidin-coated glass surface and incubated in BMDC culture medium. After $\sim 99 \%$ laser photobleaching of Cy5 fluorophore, a $102.5 \% \pm 2.4$ enhancement in Cy3 fluorescence was observed (Supplementary Fig. 32). As a negative control, when $\mathrm{Cy} 3$ and $\mathrm{Cy} 5$ fluorophores in $\mathrm{DN1} \mathbf{1}_{\text {coated }}$ were placed at distances $>$ Förster distance, no appreciable enhancement in Cy3 fluorescence was observed (Supplementary Fig. 33). Furthermore, we verified in bulk studies that $\mathrm{Cy} 3$ fluorescence enhancement could be abolished by pre-incubation with DNase I (which degrades the structure) or PEI (which crushes the structure) (Supplementary Note 2 and Supplementary Figs 5153). Interestingly, we found that even after $50 \%$ of the $\mathrm{Cy} 3$ fluorescence enhancement had been pre-degraded by DNase I, structural integrity (assessed by AGE and TEM) was retained. This is consistent with a model where DNs can sustain a large number of local strand scissions before catastrophic loss of structure occurs.

Encouraged by our cell-free results, we incubated $\mathrm{Cy} 3 / \mathrm{Cy} 5$ labelled DN1 $1_{\text {coated }}$ with BMDCs for $12 \mathrm{~h}$ and washed the cells to remove excess structures. We then monitored the enhancement of Cy 3 fluorescence upon Cy5 photobleaching at 0, 6, 12 and $24 \mathrm{~h}$ time points (Supplementary Figs $34-37$ ). In this experiment, we observed enhancement of cellular donor fluorescence of $76.13 \% \pm 8.2$ at $0 \mathrm{~h}, 69.63 \% \pm 7.3$ at $6 \mathrm{~h}, 59.27 \% \pm 8.5$ at $12 \mathrm{~h}$ and $49.14 \% \pm 9.8$ at $24 \mathrm{~h}$, respectively (Fig. 4 ).

The 20\% decrease in the enhancement of donor fluorescence at $0 \mathrm{~h}$ post washing relative to the cell-free measurement is indicative of either our inability to accurately replicate endosomal and endolysosomal environments, or that some of the $\mathrm{DN1} \mathbf{1}_{\text {coated }}$ are already partially degraded, or both. At $24 \mathrm{~h}$ post washing, $49.14 \% \pm 9.8$ enhancement of donor fluorescence was observed, suggesting that structurally intact $\mathrm{DN1} \mathbf{1}_{\text {coated }}$ are still present within cells. We note here again that our cell-free experiments (Supplementary Note 2 and Supplementary Fig. 51) demonstrated that structures that display $50 \%$ of maximum fluorescence enhancement remained globally intact as indicated by agarose-gel and TEM analysis; this implies that most particles retained their structural integrity in endosomes at our $24 \mathrm{~h}$ post-washing time point. A model consistent with these observations is that most particles that have globally collapsed are rapidly removed from the endosomal compartments; as discussed above, $\sim 87 \%$ of uptaken DNs have been cleared from endosomes by the $24 \mathrm{~h}$ post-wash time point.

Biodistribution of $\mathrm{DN1}_{\text {coated }}$ in mice. Finally, we profiled the biodistribution of Alexa 750-labelled $\mathrm{DN1} \mathbf{1}_{\text {coated }}$ injected retro-orbitally in mice and compared it with negative controls: Alexa 750-labelled DNA oligonucleotide and Alexa 750-labelled $\mathrm{DN1}_{\text {bare. }}$ Alexa 750 fluorescence was monitored every $2 \mathrm{~min}$ for $1 \mathrm{~h}$ using Perkin Elmer in vivo imaging system (IVIS, Supplementary Fig. 38). Subsequently, organs were harvested and analysed individually (Supplementary Fig. 39). Both the DNA oligonucleotide 
and the $\mathrm{DNl}_{\text {bare }}$ displayed rapid renal clearance (as evidenced by accumulation in the bladder), with a half time of $5 \mathrm{~min}$ and 9 min, respectively (Fig. 5 and Supplementary Fig. 40). In contrast, $D N 1_{\text {coated }}$ was cleared more slowly with a half-life of $\sim 45 \mathrm{~min}$; we hypothesize this improvement was due to the increased resistance of $\mathrm{DN1} \mathbf{1}_{\text {coated }}$ structures to denaturation and nuclease degradation, while in circulation.

\section{Discussion}

Herein we have developed a method that overcomes two major challenges that limit the effective utilization of DNs in vivo. Coating of DNs with positively charged oligolysine such as $\mathrm{K}_{10}$ stabilizes them against denaturation at physiological divalent-ion concentrations and remarkably results in little or no distortion of the three-dimensional structure, as assessed by inspection of negative-stain TEM images. Coating with a PEGylated oligolysine co-polymer, $\mathrm{K}_{10}-\mathrm{PEG}_{5 \mathrm{~K}}$ significantly stabilizes the DNs against nuclease degradation by $\sim 1,000$-fold. $\mathrm{K}_{10}-\mathrm{PEG}_{5 \mathrm{~K}}$-coated $\mathrm{DNs}$ are readily taken up into endosomes and endolysosomes of BMDC without any apparent cytotoxicity (that is, visible rounding of cells). A significant fraction of endocytosed DNs $(\sim 13 \%)$ persist as intact structures within the cellular compartments even after $24 \mathrm{~h}$. Preliminary mice experiments show modest improvement in circulation and biodistribution of $D N 1_{\text {coated }}$ relative to $\mathrm{DN} \mathbf{1}_{\text {bare }}$. PEGylated oligolysine therefore offers a low-cost, highly effective method for protection of DNs for local therapeutic delivery and, potentially, for systemic therapeutic delivery applications, where modest circulation times are sufficient. In the future, longer circulation times may be achievable by additional surface decorations, for example, CD47 'don't eat me' signals ${ }^{29}$.

\section{Methods \\ Materials. Tris/Borate/EDTA buffer, PCR tubes and 96-well PCR plates were purchased from VWR. Oligolysine $\left(\mathrm{K}_{4}-\mathrm{K}_{10}\right.$ and $\left.\mathrm{R}_{6}\right)$ were purchased from Peptide 2.0 as crude in a $5 \mathrm{mg}$ scale. Oligolysine $\mathrm{K}_{10}-\mathrm{PEG}_{1 \mathrm{~K}}, \mathrm{~K}_{10}-\mathrm{PEG}_{5 \mathrm{~K}}$ and $\mathrm{K}_{10}-\mathrm{PEG}_{20 \mathrm{~K}}$ were purchased from Alamada polymers in $100 \mathrm{mg}$ scale. Agarose was purchased from Lonza. Magnesium chloride, sodium chloride, glycerol, Tris base, EDTA and Tween20 was purchased from Sigma-Aldrich. RPMI, PBS, FBS and penicillin- streptomycin were purchased from Gibco. Carbon Formvar grids and uranyl for- mate were purchased from Electron Microscopy Sciences. Amicon Ultra filtration devices and 3.5 K MWCO Slide-A-Lyzer mini dialysis devices were purchased from Fisher Scientific. DNA gel extraction spin column was purchased from Bio-Rad.}

Nanostructure synthesis and purification. The design-specific staple strands were purchased from IDT Technologies in $250 \mu \mathrm{M}$ scale. The sequence of the staple strands and the design is reported in the Supplementary Information. The p7308 scaffold strand was produced from M13 phage replication in Escherichia coli and was endotoxin-purified using Triton X-114 as described previously ${ }^{13}$.

For the synthesis of DNs, $10 \mathrm{nM}$ p7308 scaffold was mixed with tenfold excess of staples in TE buffer ( $5 \mathrm{mM}$ Tris and $1 \mathrm{mM}$ EDTA) containing $10-20 \mathrm{mM} \mathrm{MgCl}_{2}$. The amount of $\mathrm{MgCl}_{2}$ varied with the structure: DN1 $(10 \mathrm{mM}), \mathrm{DN} 2(6 \mathrm{mM}), \mathrm{DN} 3$ (10 mM), DN4 (10 mM), DN5 (10 mM), DN6 (12 mM), DN7 (14 mM), DN8 $(12 \mathrm{mM})$ and DN9 $(6 \mathrm{mM})$. The solutions were subjected to a thermal annealing ramp on a Tetrad 2 Peltier thermal cycler (Bio-Rad) according to the following schedule: incubate at $65^{\circ} \mathrm{C}$ for $15 \mathrm{~min}$, decrease to $50^{\circ} \mathrm{C}$, incubate at $50^{\circ} \mathrm{C}$ for $6 \mathrm{~h}$ $30 \mathrm{~min}$ and decrease to $40{ }^{\circ} \mathrm{C}$ at $6 \mathrm{~h} 30 \mathrm{~min}^{\circ} \mathrm{C}^{-1}$. The quality of folding was analysed by AGE. Solutions of folded DN were concentrated tenfold using a $30 \mathrm{k}$ MWCO Amicon Ultra centrifugal filter device (Millipore) and then purified by glycerol gradient ultracentrifugation ${ }^{30}$. The $45 \%$ and $15 \%$ glycerol solutions were made in $1 \times$ TE buffer containing the same levels of $\mathrm{MgCl}_{2}$ as required for folding. The glycerol fractions containing nanostructures was concentrated and buffer exchanged to remove glycerol using a 30k MWCO Amicon Ultra centrifugal filter device. Following purification, the stock solution was diluted appropriately for TEM imaging to verify quality. The stock concentration was determined by ultraviolet absorbance at $260 \mathrm{~nm}$ on a Nanodrop spectrophotometer (Thermo Scientific), assuming that $A_{260}=1$ for $50 \mu \mathrm{g} \mathrm{ml}^{-1}$ DNs. Stock solutions were stored at $4{ }^{\circ} \mathrm{C}$ until use.

Agarose gel electrophoresis. After folding, DNs were analysed by AGE ( $2 \%$ agarose, $0.5 \times \mathrm{TBE} 10 \mathrm{mM} \mathrm{MgCl}_{2}, 1 \times$ ethidium bromide) using Thermo Scientific EasyCast Mini Gel System apparatus. Ten microlitres of samples were mixed with $3 \mu \mathrm{l}$ of $6 \times$ loading buffer and were loaded into the agarose gel. The samples were allowed to migrate for $2-3 \mathrm{~h}$ (running buffer: $0.5 \times$ TBE, $11 \mathrm{mM}$ $\mathrm{MgCl}_{2} ; 65 \mathrm{~V}$ ). The gel was imaged with Typhoon FLA 9000 (GE Healthcare Life Sciences). To recover nanostructures, the bands were visualized with ultraviolet light and cut out from the gel. Extracted bands were crushed and placed into a DNA gel extraction spin column (Bio-Rad). The nanostructure solution was recovered by centrifugation of the loaded column for $5 \mathrm{~min}$ at $13,000 \mathrm{~g}$.

Negative-stain TEM analysis. Three microlitres of DNs were pipetted onto a plasma-treated carbon Formvar grid (Electron Microscopy Sciences) and incubated for $1 \mathrm{~min}$. The solution was wicked away onto filter paper and $7 \mu \mathrm{l}$ of freshly prepared $2 \%$ uranyl formate (in $\mathrm{H} 2 \mathrm{O}, \mathrm{w} / \mathrm{v}$ ) was immediately added. This was incubated for $0.5 \mathrm{~min}$ and then wicked away by filter paper. Imaging was carried out on a JEOL 1400 TEM.

Coating with $\mathbf{K}_{\mathbf{1 0}}$ and $\mathbf{K}_{\mathbf{1 0}}-\mathbf{P E G}_{\mathbf{n k}}$. Ten microlitres of $10 \mathrm{nM}$ DN1-DN9 was mixed with $1 \mu \mathrm{l}$ of oligolysine $\left(\mathrm{K}_{10}\right.$ or $\left.\mathrm{K}_{10}-\mathrm{PEG}_{n \mathrm{~K}}\right)$ at a concentration where the desired N:P ratio (ratio of nitrogen in amines:phosphates in DNA) would be achieved and the sample incubated at room temperature for $30 \mathrm{~min} . \mathrm{K}_{10}-\mathrm{PEG}_{n \mathrm{~K}}$ were purchased from Alamanda polymers, their polydispersity index from gel permeation chromatography is between 1.00 and 1.20 . The number average molecular weight range by NMR as provided by the company for $\mathrm{K}_{10}-\mathrm{PEG}_{1 \mathrm{~K}}$ is $2,200-3,100 \mathrm{Da}$, for $\mathrm{K}_{10}-\mathrm{PEG}_{5 \mathrm{~K}}$ is $5,800-7,500 \mathrm{Da}$ and for $\mathrm{K}_{10}-\mathrm{PEG}_{20 \mathrm{~K}}$ is $18,000-$ $22,000 \mathrm{Da}$.

Magnesium depletion assays. Bare DNs and $\mathrm{K}_{10}$ or $\mathrm{K}_{10}-\mathrm{PEG}_{5 \mathrm{~K}}$-coated DNs were diluted in RPMI-1640 medium (Gibco) such that the final $\mathrm{Mg}^{2+}$ concentration is $0.6 \mathrm{mM}$. The samples were incubated at $37^{\circ} \mathrm{C}$ for $1 \mathrm{~h}$ on a Tetrad 2 Peltier thermal cycler (Bio-Rad) and analysed using AGE and TEM imaging.

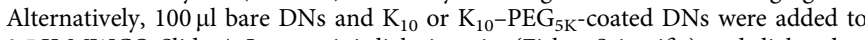
3.5 K MWCO Slide-A-Lyzer mini dialysis units (Fisher Scientific) and dialysed overnight at room temperature into zero- $\mathrm{Mg}^{2}+$ buffer ( $5 \mathrm{mM}$ Tris, $1 \mathrm{mM}$ EDTA $\mathrm{pH}$ 8.0). The liquid remaining in the dialysis unit was collected and analysed using AGE and negative-stain TEM.

Nuclease degradation assay. Bare DNs, $\mathrm{K}_{10}$ or $\mathrm{K}_{10}-\mathrm{PEG}_{5 \mathrm{~K}}$-coated DNs were diluted in RPMI-1640 medium (Gibco) containing different amounts of DNase I or $10 \%$ freshly thawed FBS (Gibco; heat-inactivated at $56^{\circ} \mathrm{C}$ by the vendor) such that the final $\mathrm{Mg}^{2+}$ ion concentration is $0.6 \mathrm{mM}$. The samples were incubated at $37^{\circ} \mathrm{C}$ for $0-24 \mathrm{~h}$ on a Tetrad 2 Peltier thermal cycler (Bio-Rad) and analysed using AGE and TEM imaging.

Nuclease deactivation assay. Freshly prepared cell medium containing 10\% FBS and solutions containing up to $50 \mathrm{U} \mathrm{ml}^{-1}$ DNase I could be inactivated by the addition of $5 \mathrm{mM}$ EGTA and $10 \% \beta$-mercaptoethanol, and incubation at $37^{\circ} \mathrm{C}$ for $30 \mathrm{~min}$.

Removal of $\mathbf{K}_{\mathbf{1 0}}$ or $\mathbf{K}_{\mathbf{1 0}}-\mathbf{P E G}_{\boldsymbol{n} \mathbf{K}}$ shell from DNs. Chondroitin sulfate ( $100 \times$, excess to the number of amines) was added to $K_{10}$ or $K_{10}-P G_{5 K}$-coated DNs and the medium adjusted to $10 \mathrm{mM} \mathrm{Mg}$. The sample was incubated at $37^{\circ} \mathrm{C}$ for 1-2 $\mathrm{h}$ during which the oligolysine shell was removed from the DNs upon electrostatic binding of oligolysine with chondroitin sulfate. In the nuclease degradation assays, nucleases were deactivated using EGTA and $\beta$-mercaptoethanol before the removal of the oligolysine shell.

In vitro microscopy measurements. Sample preparation: a 1.5 coverslip and a glass slide were sandwiched together by two strips of double-sided tape to form a flow chamber. The volume of the flow chamber was $\sim 20 \mu$ l. The chamber was incubated for $30 \mathrm{~min}$ with $20 \mu \mathrm{lof} 1 \mathrm{mg} \mathrm{ml}^{-1}$ BSA-Biotin, followed by incubation for $30 \mathrm{~min}$ with $20 \mu \mathrm{l}$ of $1 \mathrm{mg} \mathrm{ml}^{-1}$ streptavidin. The chamber was then washed three times with PBS. Twenty microlitres of $\mathrm{K}_{10}-\mathrm{PEG}_{5 \mathrm{~K}}$-coated biotinylated DNs were then introduced in the flow chamber at $10 \mathrm{nM}$ concentrations. The chamber was epoxy sealed before imaging. Imaging: samples were imaged using Zeiss Axio Observer inverted fluorescence microscope. Samples were excited either with $540-580 \mathrm{~nm}$ LED (Colibri; Cy3 excitation) or with a $625 \mathrm{~nm}$ LED (Colibri; Cy5 excitation). Zeiss filter sets $43 \mathrm{HE}$ and 50 were used for Cy3 and Cy5 imaging, respectively. Signal was collected using an Orca-Flash $4.0 \mathrm{sCMOS}$ from Hamamatsu. All data were collected using a $10 \times / 0.3 \mathrm{Ph} 1(\mathrm{WD}=5.2 \mathrm{~mm})$ Zeiss objective. Built-in image processing and analysis tools from Fiji (http://fiji.sc/Fiji) were used to analyse all data.

Isolation and culture of BMDCs. BMDCs were derived using established methods ${ }^{31}$. Briefly, bone marrow cells were isolated from female C57Bl/6J mice (Jackson Laboratories) and cultured in RPMI (Lonza) supplemented with $10 \%$ heat-inactivated FBS (Sigma-Aldrich), 1\% penicillin/streptomycin, $50 \mu \mathrm{M}$ $\beta$-mercaptoethanol (Sigma-Aldrich) and $20 \mathrm{ng} \mathrm{ml}^{-1}$ granulocyte-macrophage 
colony-stimulating factor (Peprotech). Non-adherent dendritic cells between day 7 and 10 were harvested and used for experiments.

Cell uptake assay. For uptake studies, BMDCs were seeded at a density of 50,000 cells per well into 1.5 glass-bottom 96-well plates from Mat-tek and allowed to grow overnight. Samples were prepared by diluting them to $10 \mathrm{nM}$ concentrations in a total volume of $100 \mu \mathrm{l}$ BMDC cell medium (RPMI-1640 supplemented with $10 \%$ FBS, $\beta$-mercaptoethanol and granulocyte-macrophage colony-stimulating factor). Cell uptake was monitored using confocal microscopy (SP5 $\times$ MP inverted confocal microscope, Leica) at time points between 0 and $24 \mathrm{~h}$. A sequential $z$-stack was imaged using a $0.2 \mu \mathrm{m}$ slice thickness, in the following excitation sequence: first scan: Cy3 excitation/Cy5 emission, second scan: bright field and Cy3 excitation/ Cy 3 emission; and third scan: $\mathrm{Cy} 5$ excitation/Cy5 emission. To bleach the acceptor dye, region of interest was set to $2.5 \times$ zoom of the initial field of view and laser power for Cy5 excitation was increased to $100 \%$. The slice was bleached for $10 \mathrm{~min}$, until the Cy5 signal was absent. Zoom was reset to 1 and a new $z$-stack of the initial field of view was recorded.

Cell image analysis. $Z$-stacks were loaded into Imaris software package and dimensions were cropped to represent the cell area. The new stacks were imported into image processing and analysis software from Fiji and a $Z$ projection based on average intensity was made. Regions inside and outside the bleached area were selected using the freehand selection tool and the integrated intensity was measured. DN1 containing only 18 Cy3 (donor-only) dyes or 18 Cy5 dyes (acceptor only) were used to estimate the background corrections.

Biodistribution experiments. Optical imaging experiments on mice were carried out using the IVIS spectrum instrument (Perkin Elmer). Three mice per sample were anaesthetized under $2 \%$ isofluorane and were injected retro-orbitally with $100 \mu \mathrm{l}$ volume of Alexa 750 oligo, $\mathrm{DN} \mathbf{1}_{\text {bare }}$ and $\mathrm{DN1} \mathbf{1}_{\text {coated }}$ functionalized with 36 Alexa 750 fluorophores such that final concentration of Alexa 750 fluorophore is $0.625 \mu \mathrm{M}$. The mice were immediately transferred to the imaging system and maintained at $2 \%$ isofluorane throughout imaging. Fluorescence images for kinetic analysis were acquired by a $30 \mathrm{~s}$ excitation at $745 \mathrm{~nm}$ and emission at $800 \mathrm{~nm}$, every $2 \mathrm{~min}$ for a total of $1 \mathrm{~h}$ post injection. After collection of fluorescence data, the mice were immediately killed, and blood, urine and organs were harvested and were imaged on the IVIS system. Analysis was carried out on ImageJ. Fluorescence accumulation in the bladder was measured by using the freehand selection tool to draw a region of interest around the bladder and then integrating the density. The data set was normalized to the maximum fluorescence intensity in the bladder, when Alexa 750 dye oligo is administered alone. Fluorescence accumulation in bladder versus time was fitted to an exponential using MATLAB to extract half-life.

Animal use. All animal studies were performed in accordance with NIH guidelines, under the approval of Harvard University's Institutional Animal Care and Use Committee.

Data availability. Data supporting the findings of this study are available within the article (and its Supplementary Information files) and from the corresponding author upon reasonable request.

\section{References}

1. Rothemund, P. W. K. Folding DNA to create nanoscale shapes and patterns. Nature 440, 297-302 (2006)

2. Douglas, S. M. et al. Self-assembly of DNA into nanoscale three-dimensional shapes. Nature 459, 414-418 (2009).

3. Dietz, H., Douglas, S. M. \& Shih, W. M. Folding DNA into twisted and curved nano-scale shapes. Science 325, 725-730 (2009).

4. Han, D. et al. DNA origami with complex curvatures in three-dimensional space. Science 332, 342-346 (2011).

5. Wei, B., Dai, M. \& Yin, P. Complex shapes self-assembled from single-stranded DNA tiles. Nature 485, 623-626 (2012).

6. Ke, Y., Ong, L. L., Shih, W. M. \& Yin, P. Three-dimensional structures self-assembled from DNA bricks. Science 338, 1177-1183 (2012).

7. Li, J. et al. Self-assembled multivalent DNA nanostructures for noninvasive intracellular delivery of immunostimulatory CpG oligonucleotides. ACS Nano 5, 8783-8789 (2011).

8. Andersen, E. S. et al. Self-assembly of a nanoscale DNA box with a controllable lid. Nature 459, 73-76 (2009).

9. Douglas, S. M., Bachelet, I. \& Church, G. M. A logic-gated nanorobot for targeted transport of molecular payloads. Science 335, 831-834 (2012).

10. Liu, M. et al. A DNA tweezer-actuated enzyme nanoreactor. Nat. Commun. 4 , 2127 (2013).

11. Banerjee, A. et al. Controlled release of encapsulated cargo from a DNA icosahedron using a chemical trigger. Angew. Chem. Int. Ed. 52, 6854-6857 (2013).
12. Jahnen-Dechent, W. \& Ketteler, M. Magnesium basics. Clin. Kidney J. 5, i3-i14 (2012).

13. Hahn, J., Wickham, S. F. J., Shih, W. M. \& Perrault, S. D. Addressing the instability of DNA nanostructures in tissue culture. ACS Nano 8, 8765-8775 (2014).

14. Conway, J. W., McLaughlin, C. K., Castor, K. J. \& Sleiman, H. DNA nanostructure serum stability: greater than the sum of its parts. Chem. Commun. 49, 1172-1174 (2013).

15. Mei, Q. et al. Stability of DNA origami nanoarrays in cell lysate. Nano Lett. 11, 1477-1482 (2011).

16. Surana, S., Bhatia, D. \& Krishnan, Y. A. Method to study in vivo stability of DNA nanostructures. Methods 64, 94-100 (2013).

17. Cassinelli, V. et al. One-step formation of chain-armour stabilized DNA nanostructures. Angew. Chem. Int. Ed. 54, 7795-7798 (2015).

18. Benson, E. et al. DNA rendering of polyhedral meshes at the nanoscale. Nature 523, 441-444 (2015).

19. Veneziano, R. et al. Designer nanoscale DNA assemblies programmed from the top down. Science 352, 1534 (2016).

20. Mikkilä, J. et al. Virus-encapulated DNA origami nanostructures for cellular delivery. Nano Lett. 14, 2196-2200 (2014).

21. Kiviaho, J. K. et al. Cationic polymers for DNA origami coating - examining their binding efficiency and tuning the enzymatic reaction rates. Nanoscale $\mathbf{8}$, 11674-11680 (2016).

22. Perrault, S. D. \& Shih, W. M. Virus-inspired membrance encapsulation of DNA nanostructures to achieve in vivo stability. ACS Nano 8, 5132-5140 (2014).

23. Boussif, O. et al. A versatile vector for gene and oligonucleotide transfer into cells in culture and in vivo: polyethyleneimine. Proc. Natl Acad. Sci. USA 92, 7297-7301 (1995).

24. van Vlerken, L. E., Vyas, T. K. \& Amiji, M. M. Poly(ethylene glycol)-modified nanocarriers for tumor-targeted and intracellular delivery. Pharm. Res. 24, 1405-1414 (2007).

25. Vanecko, S. \& Laskowski, Sr M. Studies of the specificity of deoxyribonuclease I. III. Hydrolysis of chains carrying a monoesterified phosphate on carbon $5^{\prime}$. J. Biol. Chem. 236, 3312-3316 (1961).

26. Haubner, R. et al. Structural and functional aspects of RGD-containing cyclic pentapeptides as highly potent and selective integrin $\alpha \mathrm{v} \beta 3$ antagonists. J. Am. Chem. Soc. 118, 7461-7472 (1996).

27. Yoshinaga, T., Yasuda, K., Ogawa, Y. \& Takakura, Y. Efficient uptake and rapid degradation of plasmid DNA by murine dendritic cells via a specific mechanism. Biochem. Biophys. Res. Commun. 299, 389-394 (2002).

28. Mekler, V. M. A photochemical technique to enhance sensitivity of detection of fluorescence resonance energy transfer. Photochem. Photobiol. 59, 615-620 (1994).

29. Rodriguez, P. L. et al. Minimal 'self peptides that inhibit phagocytic clearance and enhance delivery of nanoparticles. Science 339, 971-975 (2013).

30. Lin, C., Perrault, S. D., Kwak, M., Graf, F. \& Shih, W. M. Purification of DNA-origami nanostructures by rate-zonal centrifugation. Nucleic Acids Res. 41, e40 (2013).

31. Bhattacharya, P., Gopisetty, A., Ganesh, B. B., Sheng, J. R. \& Prabhakar, B. S. GM-CSF-induced, bone-marrow-derived dendritic cells can expand natural Tregs and induce adaptive Tregs by different mechanisms. J. Leukoc. Biol. 89, 235-249 (2011).

32. Douglas, S. M. et al. Rapid prototyping of 3D DNA-origami shapes with caDNAno. Nucleic Acids Res. 37, 5001-5006 (2009).

\section{Acknowledgements}

We are grateful to Schlumberger Faculty for Future Fellowship support to N.P., Human Frontiers of Science Foundation Fellowship support to M.M.C.B., support from NSF Expeditions (1317694), NSF DMREF (1435964), Army Research Office (W911NF-12-1-0420), Dana Farber Cancer Institute Claudia Adams Barr Award and the Wyss Institute at Harvard Faculty Award to W.M.S. We thank Garry Cuneo and Thomas Ferrante for maintaining the TEM and microscopy facilities.

\section{Author contributions}

N.P., M.M.C.B. and W.M.S. designed the experiments. N.P. completed the experiments M.M.C.B. performed the cellular uptake experiments. M.V. designed the FRET system. B.N. performed the in vitro FRET experiments. J.H.R., L.Y.T.C. and F.M.A. performed the cyclic arginine-glycine-aspartate uptake study. W.A.L. and M.M.C.B. performed the biodistribution experiments. N.P., M.M.C.B., B.N., D.J.M. and W.M.S. wrote the paper

\section{Additional information}

Supplementary Information accompanies this paper at http://www.nature.com/ naturecommunications 
Competing interests: N.P., M.M.C.B. and W.M.S. have filed a patent

(PCT/US2014/064659) covering positive amine-induced protection of DNs from in vivo threats. The remaining authors declare no competing financial interests.

Reprints and permission information is available online at http://npg.nature.com/ reprintsandpermissions/

How to cite this article: Ponnuswamy, N. et al. Oligolysine-based coating protects DNA nanostructures from low-salt denaturation and nuclease degradation. Nat. Commun. 8, 15654 doi: 10.1038/ncomms15654 (2017).

Publisher's note: Springer Nature remains neutral with regard to jurisdictional claims in published maps and institutional affiliations. (c) Open Access This article is licensed under a Creative Commons Attribution 4.0 International License, which permits use, sharing, adaptation, distribution and reproduction in any medium or format, as long as you give appropriate credit to the original author(s) and the source, provide a link to the Creative Commons license, and indicate if changes were made. The images or other third party material in this article are included in the article's Creative Commons license, unless indicated otherwise in a credit line to the material. If material is not included in the article's Creative Commons license and your intended use is not permitted by statutory regulation or exceeds the permitted use, you will need to obtain permission directly from the copyright holder. To view a copy of this license, visit http://creativecommons.org/ licenses/by/4.0/

(C) The Author(s) 2017 